\title{
Editorial
}

\section{Traditional Meat Products: Improvement of Quality and Safety}

\author{
Marta Laranjo,, ${ }^{1,2}$ Régine Talon, ${ }^{3}$ Andrea Lauková, ${ }^{4}$ Maria J. Fraqueza, ${ }^{5}$ and Miguel Elias ${ }^{1,6}$ \\ ${ }^{1}$ Instituto de Ciências Agrárias e Ambientais Mediterrânicas (ICAAM), Universidade de Évora, Pólo da Mitra, Ap. 94, \\ 7002-554 Évora, Portugal \\ ${ }^{2}$ Instituto de Investigação e Formação Avançada (IIFA), Universidade de Évora, Évora, Portugal \\ ${ }^{3}$ Université Clermont Auvergne, INRA, MEDIS, Clermont-Ferrand, France \\ ${ }^{4}$ Institute of Animal Physiology, Slovak Academy of Sciences, Košice, Slovakia \\ ${ }^{5}$ CIISA, Faculty of Veterinary Medicine, University of Lisbon, Avenida da Universidade Técnica, Pólo Universitário do Alto da Ajuda, \\ 1300-477 Lisbon, Portugal \\ ${ }^{6}$ Departamento de Fitotecnia, Escola de Ciências e Tecnologia, Universidade de Évora, Pólo da Mitra, Ap. 94, 7002-554 Évora, Portugal
}

Correspondence should be addressed to Marta Laranjo; mlaranjo@uevora.pt

Received 6 November 2017; Accepted 7 November 2017; Published 21 November 2017

Copyright (C) 2017 Marta Laranjo et al. This is an open access article distributed under the Creative Commons Attribution License, which permits unrestricted use, distribution, and reproduction in any medium, provided the original work is properly cited.

What are traditional meat products? Traditional meat products are high sensory quality foods, usually with high nutritional value, produced in a small scale, using ingredients and procedures from ancient times. Producers must have the ability to satisfy the expectations of consumers, regarding sensory, nutritional aspects and safety, considering that they are increasingly more demanding and more informed, turning this into an important challenge. Usually, a meat product is defined as foods that consist of or contain meat. The flesh of an animal, typically a mammal or bird, is considered as meat. However, fish meat is also present in our diet and several traditional processed products come from it. Thus, traditional meat products sensu lato include food products derived from both meat and fish meat.

All over the world, different processes are used to preserve meat and the particular know-how of people living in different regions gave rise to a great diversity of meat products according to their traditions and historic use. These traditional processes, their particularities, and their effect on the quality and safety of meat products are important research topics. The scope of this edition was to disseminate high-quality research related with traditional meat products and review recent developments on the quality and safety improvement of traditional meat products worldwide, such as the use of starter cultures or the application of new preservation methods.

Among the 16 submitted manuscripts, six have been selected to be part of this special issue.
M. Trevisani et al. studied the effect of beeswax coating of foods on the oxidative stability of Italian salami. Beeswax coating constitutes a barrier to oxygen, light, and vapour, which may prevent oxidation of fat and pigments and water loss. Furthermore, the authors concluded that beeswax coating prevents case hardening and facilitated the peeling.

T. Yin et al. have evaluated the quality of silver carp (Hypophthalmichthys molitrix) surimi (SCS) gels incorporated with fish bone and found out that size reduction of the fish bone improved the quality of the SCS gel maintaining better gel matrices.

M. Chmiel et al. have studied two types of kabanosy sausages, Traditional Specialties Guaranteed (TSG), from Poland and concluded that their drying process could be shortened without compromising the authenticity of the products and with the concomitant advantages to producers.

R. Zhang et al. investigated the effects of different ozone treatments on the physicochemical characteristics of myofibrillar proteins from silver carp (H. molitrix) surimi during frozen storage.

A. Holck et al. reviewed the possible health effects of the ingredients used in fermented sausages. Recent attempts to improve sausages from the nutritional point of view include partial replacement of saturated by unsaturated fats, reducing the use of sodium chloride or replacement by potassium chloride, and the use of selected starter cultures. They also review the processing and postprocessing strategies to inhibit the growth of food pathogenic microorganisms, such as 
Escherichia coli, Salmonella enterica, Staphylococcus aureus, Listeria monocytogenes, Clostridium botulinum, and Toxoplasma gondii, and reduce their presence in the products.

The paper "The Use of Starter Cultures in Traditional Meat Products" by M. Laranjo et al. reviews the role and mode of action of bacterial and fungal starter microbiota, focusing on the development of starter cultures better adapted to the meat matrix. Furthermore, omics approaches on starter cultures are revised, because the use of these techniques allows rapid screening of strains for desirable functional characteristics.

Submitting authors come from five different countries, four European (Italy, Poland, Norway, and Portugal) and one non-European, China.

We are pleased to introduce this special issue, which includes six papers that provide new insights on the manufacturing and processing of traditional meat products sensu lato and we wish that the readers of this journal find this issue of relevance and importance to their research.

\section{Acknowledgments}

We thank the authors of the manuscripts for their contributions, as well as all the anonymous reviewers for their valuable participation in the evaluation process. M. Laranjo acknowledges a Post-Doc research grant from Fundação para a Ciência e a Tecnologia (FCT) (SFRH/BPD/108802/2015).

Marta Laranjo Régine Talon Andrea Lauková Maria J. Fraqueza Miguel Elias 

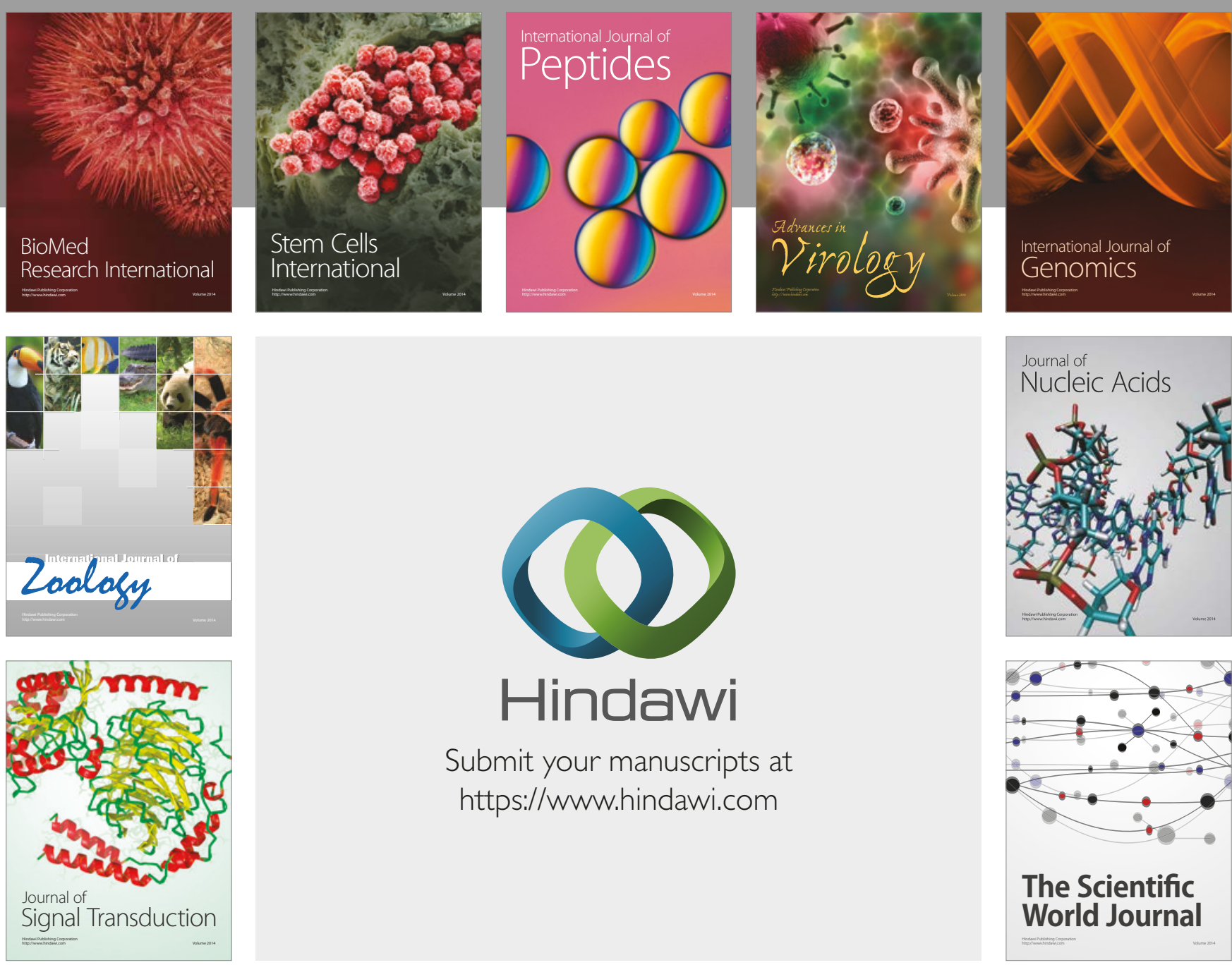

Submit your manuscripts at

https://www.hindawi.com
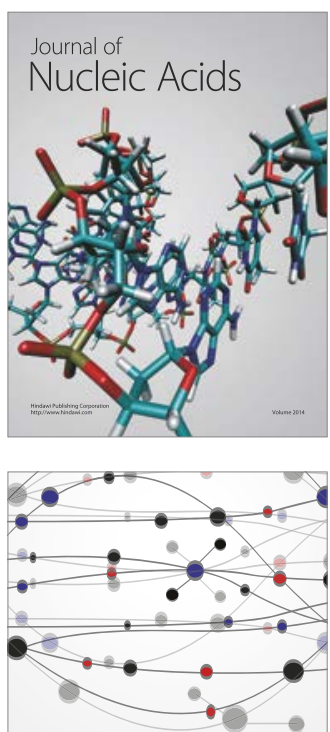

The Scientific World Journal

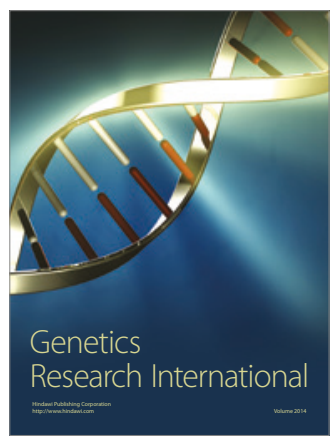

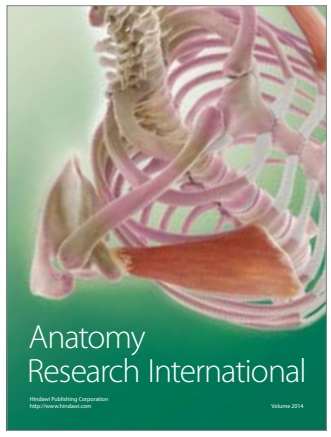

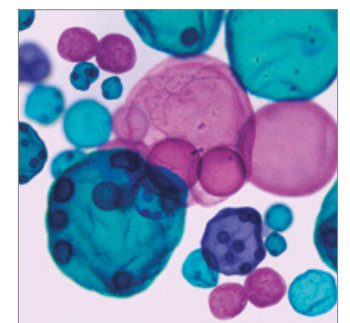

International Journal of Microbiology
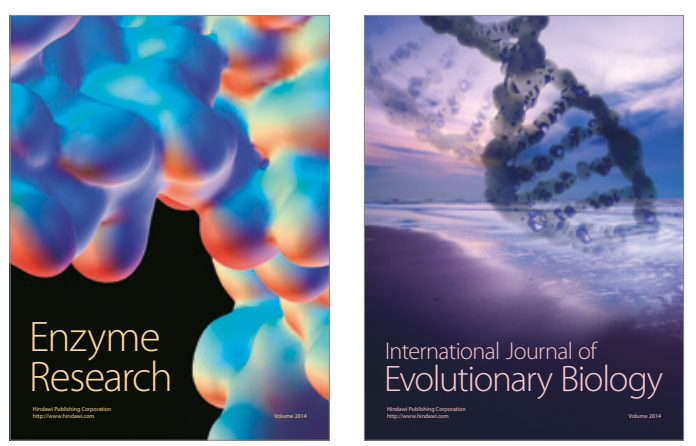
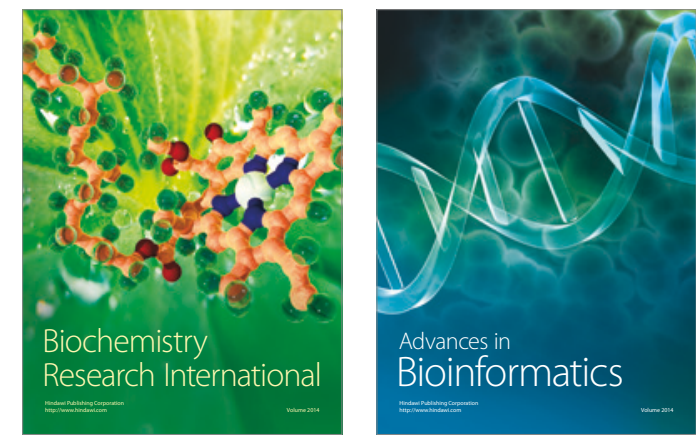

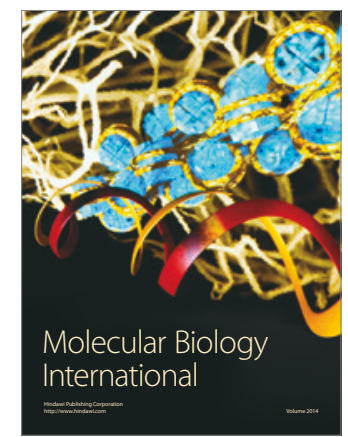

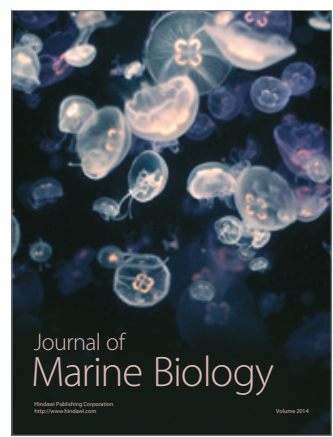

